\title{
Managing Control Measures According to the ISO 22000:2018 Requirements: the COMECAT Method
}

\author{
Y. Politis, F.D. Krokos, I. Papadakis and G. Chatzistelios
}

School of Science and Technology, Hellenic Open University Parodos Aristotelous 18, GR26 335, Patra, Greece

DOI - http://doi.org/10.37502/IJSMR.2022.5101

\begin{abstract}
Since its introduction in 2005, ISO 22000 has required a systematic approach for the selection and categorization of control measures. Given that the ISO 22000 standard did not describe a distinct method to categorize these measures and the lack of relevant methodologies in the literature, the COMECAT method was introduced in 2017 with the aim to provide clear guidance for their selection and categorization. The new version of the ISO 22000 standard, introduced in 2018, gives more insights about the categorization of control measures and, therefore, the purpose of this paper is to verify whether the COMECAT method fully conforms to the new version's requirements. The paper analyses one by one the requirements of the new ISO 22000 standard regarding the categorization of control measures and describes how the COMECAT method conforms to these requirements. The COMECAT method fully conforms to the requirements of the new version of the ISO 22000 standard and thus can be utilized by food business operators as a valuable tool for categorizing the selected identified control measure(s).
\end{abstract}

Keywords: Control measures, Categorization, Food Safety Management Systems, ISO 22000, and HACCP.

\section{Introduction}

For several decades implementation of Hazard Analysis and Critical Control Points (HACCP) methodology [1] has been quite common in food industry to safeguard consumer protection related to food safety. The use of HACCP was considerably increased when relevant requirements were introduced in European Legislation (e.g., [2]).

In 2005, ISO published its first international standard that specified requirements for a Food Safety Management System (FSMS) [3]. Since then, ISO 22000 has exhibited a worldwide implementation and the number of certificates to this standard has steadily increased during all these years. According to the ISO annual survey [4], of the number of valid certificates to ISO management system standards worldwide, a total of 33.502 ISO 22000 certificates were recorded globally in 2019, as compared to 4.122 during 2007. 
A number of studies has examined motivations and benefits as well as challenges and constraints in the implementation of Food Safety Management Systems [5-6] and highlighted the need for tools to help the food business operators to diagnose and improve their FSMS. This is especially so for small and medium-sized enterprises, as they do not always have the necessary skills (e.g., expertise), experience and/or resources (e.g., financial and staff capabilities) [7]. As demonstrated by Dzwolak [8] one of the most problematic areas of FSMS were associated with OPRPs identification.

One of the innovations of the ISO 22000 standard was the introduction of the term OPRPs (Operational Prerequisite Programs). The OPRP is a type of control measure that falls between CCPs (Critical Control Points) and PRPs (Prerequisite Programs), which already existed in the HACCP methodology (the PRPs were called Prerequisites).

The differentiation between CCPs and PRPs in the HACCP methodology was relatively simple and it was performed with the use of the traditional HACCP decision tree [9]. The introduction of the OPRPs created quite some discussion. The ISO 22000 standard did not describe a distinct method of categorization of control measures but only prescribed general criteria. As a result, quite a few methodologies appeared in the literature [10-12] and often confused users. This is especial the case for small and medium - sized enterprises, which demonstrate insufficient understanding of the relevant safety concepts, exhibiting an over reliance on the use of CCPs to control hazards in situations in which other control measures would be more appropriate [13]. In a similar way, Chen et al. [14] question the ability of organizations to implement control measures effectively and analyzed the "inspectionacceptance" of the raw materials step, which can be controlled as CCP or OPRP, according to different researchers.

The COMECAT (Control Measures Categorization) method was introduced in 2017 [15] and aimed to provide clear guidance for the selection and categorization of control measures in the framework of the implementation of a FSMS in conformance to the ISO 22000 standard.

This paper aims to verify that the COMECAT method fully conforms to the requirements of the new version of the standard [16] and thus can be utilized by food business operators as a valuable tool for categorizing the selected identified control measure(s) to be managed as $\operatorname{OPRP}(\mathrm{s})$ or as $\mathrm{CCP}(\mathrm{s})$.

As a start, the major changes in the new ISO 22000 standard are described, followed by a short description of the COMECAT method. The main discussion includes a detailed verification of how the COMECAT method conforms to all the requirements of the ISO 22000:2018 standard related to the selection and categorization of control measures including relevant examples for different types of control measures.

\section{Materials and methods}

\subsection{ISO 22000 requirements for the categorization of control measures}

ISO 22000, when first published (ISO, 2005), introduced the concept of operational prerequisite programme (OPRP) and the requirement for those control measures selected to 
control "significant" hazards, to be managed through OPRP(s) or by the HACCP plan. Furthermore, and according to the standard, the selection and categorization of control measures to OPRPs / HACCP plan shall be carried out using a logical approach that includes assessments regarding the following:

a. Its effect on identified food safety hazards relative to the strictness applied

b. Its feasibility for monitoring (e.g. ability to be monitored in a timely manner to enable immediate corrections)

c. Its place within the system relative to other control measures

d. the likelihood of failure in the functioning of a control measure or significant processing variability

e. The severity of the consequence(s) in the case of failure in its functioning

f. Whether the control measure is specifically established and applied to eliminate or significantly reduce the level of hazard(s)

g. Synergistic effects (i.e. interaction that occurs between two or more measures resulting in their combined effect being higher than the sum of their individual effects).

As the aforementioned "novelties" of ISO 22000 did not originate from the well-established CODEX HACCP principles, they have led to confusion and difficulties during implementation [8].

Regarding the categorization of control measure, the current edition of ISO 22000 [16] does not differentiate significantly from the previous standard, ISO 22000:2005. It still requires a systematic approach to be implemented and for this, it provides a series of elements that need to be considered. These elements are presented in detail and discussed in the next Section.

Although there are no significant differences between the two editions of the standard, for the categorization of control measures, ISO 22000:2018 describes the elements that need to be considered in a clearer and more structured way and thus it provides a better understanding for the development of valid methodologies.

It is now clear that all elements that need to be assessed refer exclusively to categorization and not to selection and categorization of control measures, as was the case in ISO 22000:2005.

Furthermore, there is a clear distinction between elements related to the level of risk associated with the functioning of the control measure and those related to the requirements for monitoring CCPs and/or OPRPs (feasibility of monitoring).

Apart from that, ISO 22000:2018, provides a clearer and more focused definition of the terms "operational prerequisite programme (OPRP)" and "critical control point (CCP)", in line with their basic characteristics and in consistency with the standard operating procedures of effective food safety management systems [13]. 
For example,

- OPRP is now defined as "control measure or combination of control measures applied to prevent or reduce a significant food safety hazard to an acceptable level, and where action criterion and measurement or observation enable effective control of the process and/or product" [16] and not as "PRP identified by the hazard analysis as essential in order to control the likelihood of introducing food safety hazards to and/or the contamination or proliferation of food safety hazards in the product(s) or in the processing environment" [3], whilst

- CCP is defined as " step in the process at which control measure(s) is (are) applied to prevent or reduce a significant food safety hazard to an acceptable level, and defined critical limit(s) and measurement enable the application of corrections" [16] as compared to " step at which control can be applied and is essential to prevent or eliminate a food safety hazard or reduce it to an acceptable level" [3].

As it can be seen, for CCPs, the requirement for monitoring (defined critical limits and effective measurement) that will enable on time corrections are now incorporated on the definition. For OPRPs, it is now clarified that they are not just a category of PRPs but control measures with certain target (prevent or reduce a significant food safety hazard to an acceptable level) and characteristics (action criteria, measurement or observation) very similar to the ones of a CCP.

Consequently, any systematic approach developed for the categorization of control measures to conform to ISO 22000:2018 requirements, needs to address the aforementioned elements.

\subsection{Description of the COMECAT method}

During 2017, before ISO 22000:2018 was published, Politis et al. [15] introduced COMECAT method, a method for categorization (CAT) of Control (CO) Measures (ME) in a Food Safety Management System. Until then, no other justified methodology existed for the categorization of control measures in Food Safety Management Systems. The COMECAT method attempts to help organizations deploy their limited resources to control only a few hazards of high risk as CCPs leaving as many hazards as possible to be controlled as PRPs and OPRPs. COMECAT method, which is presented in Fig. 1, utilizes the risk-based approach followed by five questions which constitute a decision tree model.

When implementing COMECAT method, first a thorough examination of the process under question is necessary to identify every hazard in each step of it. For each food safety hazard identified, its acceptable level must be defined. The use of relevant scientific publications combined with legislation and experience (best practice) is proved to be of great help in this step. 


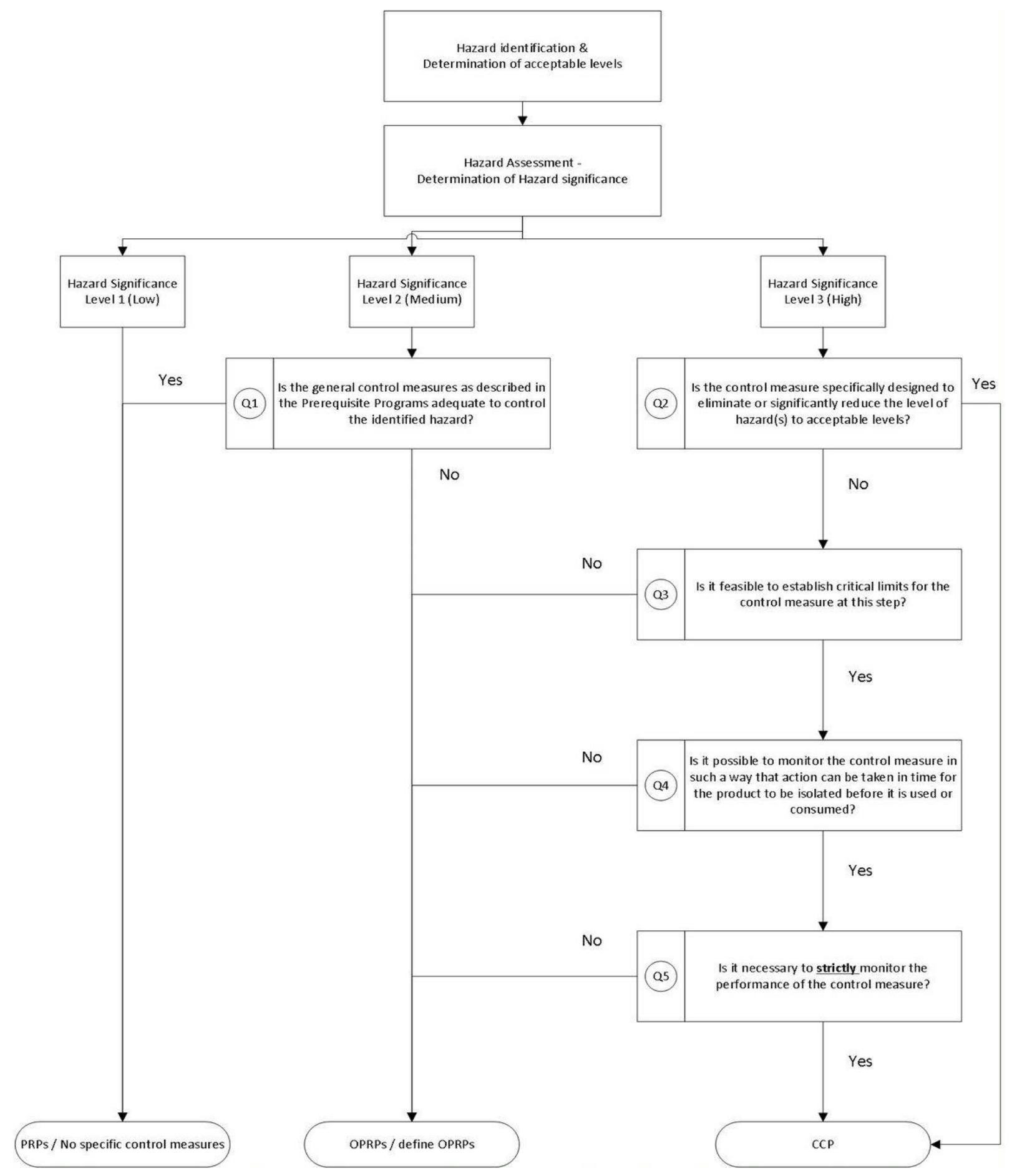

\section{Fig. 1: The COMECAT method [15]}

In the second step of COMECAT method (Hazard Assessment), the risk level of each hazard identified in the previous step must be determined. Assuming that PRPs are already correctly implemented and considering the next steps of the process where an elimination or reduction to an acceptable risk level is possible, the risk level is defined by the severity of adverse health effects of the hazard in relation to their occurrence probability in the end product, if the considered (specific) control measures fail or are not present. Qualitative scales for severity and likelihood can be used in the face of hazard assessment, as presented in Table 1. 
In this way, three hazard significance levels can be defined for each of the identified food hazards. The hazard assessment performed for each identified hazard results in either one of three specified significance levels:

- Level 1 (hazards of low significance) that should be controlled as PRPs

- Level 2 (hazards of medium significance) that should be controlled as OPRPs unless PRPs are adequate to control the identified hazard

- Level 3 (hazards of high significance) that should be further examined through the decision tree model to categorize control measures as OPRPs or CCPs

According to COMECAT's decision tree model, a hazard of high significance is then thoroughly examined through a series of four questions (questions Q2 to Q5 in Fig. 1). Depending on the answer given on each question, control measures in place are either categorized as a CCP or as an OPRP.

More specifically, if answering Q2 with a "yes", which means that the control measure is specifically designed to eliminate or significantly reduce the level of hazard(s) to acceptable levels, this implies that all characteristics of a CCP, such as measurable critical limits, in time monitoring, etc, exist. Otherwise, if the answer to Q2 is "no" the method continues with the next question.

In Q3 the feasibility of establishing Critical Limits to the control measure is examined. Critical Limits are used to separate acceptable from unacceptable conditions. If Critical Limits can be established, then the method continues with the next question otherwise the control measure should be regarded as OPRP. Q4 examines the possibility of monitoring the control measure in such a way that action when Critical Limits are exceeded can be taken in time for the product to be isolated before it is used or consumed. If this is not possible or necessary (if for example loss of control does not mean necessarily the provision of unsafe products), OPRP(s) can be used as control measure(s). Otherwise, the method proceeds to the final question Q5.

In Q5, which is the last question of the decision tree, the necessity for a strict monitoring of the performance of the specific control measure is evaluated. For this, evaluation factors such as synergistic effects and the stability of the performance of the control measure should be examined. If strict monitoring of the performance of the specific control measure is considered necessary, then the control measure can be categorized as a $\mathrm{CCP}$, otherwise as OPRP.

\section{Results and discussion}

ISO 22000:2018 requires from organizations that have implemented the standard to select an appropriate control measure or combination of control measures that will be capable of preventing or reducing the identified significant food safety hazards to defined acceptable levels. They need to categorize the selected identified control measure(s) to be managed as OPRP(s) or as CCP(s) and they must follow a systematic approach to do so. This section describes how the COMECAT method conforms to the requirements of the new standard regarding the categorization of the control measures. 
Politis et al. [15] proved the applicability of the method by implementing it to feta cheese production. The examples presented in Table $\mathrm{X}$ of the appendix refer to this application.

According to ISO 22000:2018, the categorization shall be carried out using a systematic approach. For each of the control measures selected, there shall be an assessment of the following [16]:

a) The likelihood of failure of its functioning

b) The severity of the consequence in the case of failure of its functioning; this assessment shall include:

1) The effect on identified significant food safety hazards

2) The location in relation to other control measure(s)

3) Whether it is specifically established and applied to reduce the hazards to an acceptable level

4) Whether it is a single measure or is part of combination of control measure(s).

\begin{tabular}{|c|c|c|c|c|c|}
\hline \multirow{4}{*}{ 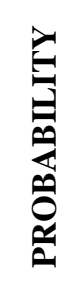 } & High 4 & & & & \\
\hline & Real 3 & & & & \\
\hline & Small 2 & & & & \\
\hline & Very small 1 & & & & \\
\hline \multicolumn{2}{|c|}{$\begin{array}{l}\text { Hazard Significance } \\
\text { Level } 1 \text { (Low) }\end{array}$} & \multirow{4}{*}{$\begin{array}{c}\text { Limited } \\
1\end{array}$} & \multirow{4}{*}{$\begin{array}{c}\text { Moderate } \\
2\end{array}$} & \multirow{4}{*}{$\begin{array}{c}\text { Serious } \\
3\end{array}$} & \multirow{4}{*}{$\begin{array}{c}\text { Very Serious } \\
4\end{array}$} \\
\hline Haza & Significance & & & & \\
\hline Leve & (Medium) & & & & \\
\hline \multicolumn{2}{|c|}{$\begin{array}{l}\text { Hazard Significance } \\
\text { Level } 1 \text { (High) }\end{array}$} & & & & \\
\hline & & \multicolumn{4}{|c|}{ EFFECT } \\
\hline
\end{tabular}

Table 1: Risk analysis matrix (adopted from Commission Notice [17])

As described in detail in the previous Section, COMECAT method is based on hazard assessment, through a risk analysis matrix, followed by a five-question systematic approach to categorize the identified control measures. PRPs are chosen to control low significance hazards, while OPRPs and CCPs are selected for medium and high significance hazards, respectively, further considering the specific characteristics of the control measures. Furthermore, the COMECAT method clearly states that when evaluating the required performance of the control measure to provide safe foods, organizations should take into consideration the stability (Table X.1) of the performance of the control measure (e.g., likelihood of failure of its functioning or significant processing variability). Where objective evidence (e.g., historical data, literature, and validation data) shows that the control measure is delivering a significantly more stringent control of the hazard than needed, the control measure can be categorized as OPRP. 
The COMECAT methodology considers the effect of the control measure to the identified significant food safety hazard as well as the location of the control measure in relation to others (Table X.2 and X.3 respectively).

Moreover, control measures that are specifically designed to eliminate or significantly reduce the level of hazard(s) to acceptable levels are treated as CCPs (Table X.4).

When categorizing control measures, the COMECAT method, also evaluates their potential synergistic effects (Table X.5). For example, the combination of microbiological control measures may be more efficient when it is multi-targeted, that is, when various individual measures are selected so that different factors effecting microbial survival are targeted.

ISO 22000:2018 further requires that the systematic approach used to categorize each one of the control measures must include an assessment of the feasibility of [16]:

a. Establishing measurable critical limits and/or measurable/observable action criteria

b. Monitoring to detect any failure to remain within critical limit and/or measurable/observable action criteria

c. Applying timely corrections in case of failure.

The COMECAT method applies specific questions to categorize control measures in relation to the aforementioned requirements. According to the methodology, a control measure is characterized as CCP only if it has specific measurable Critical Limits, which can separate acceptable from unacceptable conditions and only if corrective actions can be implemented without hesitation when monitoring indicates a deviation from the Critical Limit (Table X.6). If it is not feasible to establish Critical Limits for the control measure, OPRP(s) can be used instead.

These are in accordance with the definitions of CCPs and OPRPs as described in the ISO 22000:2018 standard. According to these definitions, CCPs need to have defined Critical Limits and measurements that enable the application of corrections while OPRPs need action criteria and measurements or observations that enable effective control of the process and/or product. Moreover, according to the methodology, a control measure is characterized as CCP only if it is possible to be monitored in such a way that action can be taken in time for the product to be isolated before it is used or consumed (question Q4 of COMECAT). If this is not possible or necessary (in case(s)) that loss of control does not mean necessarily the provision of unsafe products), the COMECAT method proposes that OPRP(s) can be used instead as control measure(s).

\section{Conclusion}

Publication of ISO 22000:2005 standard set requirements for a Food Safety Management System at an international level. Until then, consumer protection related to food safety was implemented by companies operating in the food chain through Hazard Analysis and Critical Control Points methodology. Although ISO 22000:2005 forced these companies to adopt a systematic approach for the selection and categorization of control measures it did not provide a distinct method. 
For more than a decade companies operating in the food chain that adopted ISO 22000:2005 requirements had to improvise each time they had to select and categorize control measures for their processes. In 2017 the COMECAT method was introduced by Politis et al. [15]. COMECAT method provided an innovative and robust methodology for the selection and categorization of control measures. Its applicability and compatibility with ISO 22000:2005 were validated through its application in a case study in feta cheese production presented by Politis et al. [15].

In 2018 the Second Edition of ISO 22000 [16] gave more insights about the categorization of control measures. More specifically, according to ISO 22000:2018, the categorization of control measures shall be carried out using a systematic approach which assesses the likelihood of failure of its functioning and the severity of the consequence in the case of failure of its functioning. In this paper a detailed analysis of the ISO 22000:2018 requirements regarding the categorization of control measures and how they are met with the relevant steps of COMECAT method was presented. It was proved that the COMECAT method fully conforms to the new requirements of the ISO 22000:2018 standard and thus can be utilized by companies operating in the food chain as a valuable tool for categorizing the selected identified control measure(s).

Future research may include the implementation of the COMECAT method in different food sectors in order to verify its robustness and provide useful results to interested organizations, as well as to compare the outcomes of the methodology with HACCP plans or other attempts to categorize control measures presented in the literature.

\section{References}

1) Alimentarius Codex (1969). Hazard analysis and Critical Control Point (HACCP) system and guidelines for Its application. Annex to CAC/RCP 1-1969, Rev. 1-1969.

2) Commission Regulation EC. (2004). No. 852/2004 of 29 April 2004 on the hygiene of foodstuffs. The Official Journal of the European Union. L139 (30): 1-54.

3) ISO (2005). ISO 22000:2005, Food Safety Management Systems - Requirements for any Organization in the Food Chain. International Organization for Standardization.

4) ISO (2020). The ISO Survey 2019. URL: https://www.iso.org/the-iso-survey.html. Accessed 12 November 2020.

5) Escanciano C., Santos-Vijande M. (2014). Reasons and constraints to implementing an ISO 22000 food safety management system: Evidence from Spain. Food Control. 40: 50-57. [DOI: 10.1016/j.foodcont.2013.11.032]

6) Chen E., Flint S., Perry P., Perry M., Lau R. (2015). Implementation of nonregulatory food safety management schemes in New Zealand: A survey of the food and beverage industry. Food Control. 47: 569-576. [DOI: 10.1016/j.foodcont.2014.08.009]

7) Teixeira S., Sampaio P. (2013). Food safety management system implementation and certification: survey results. Total Quality Management \& Business Excellence. 24(34): 275-293. [DOI: 10.1080/14783363.2012.669556] 
8) Dzwolak W. (2019). Assessment of HACCP plans in standardized food safety management systems - The case of small-sized Polish food businesses. Food Control. 10: article 106716. [DOI: 10.1016/j.foodcont.2019.106716]

9) Alimentarius Codex (2003). Hazard analysis and Critical Control Point (HACCP) system and guidelines for Its application. Annex to CAC/RCP 1-1969, Rev. 4-2003.

10) Arvanitoyannis I.S. (2009). HACCP and ISO 22000: Application to Foods of Animal Origin. John Wiley \& Sons, West Sussex.

11) Mueller B. (2014). HACCP: Identification of CCP, CP, PRP, oPRP in the standards IFS, BRC, ISO 22000 - practical examples. URL: http://safefood-online.de/en/. Accessed 4 October 2020.

12) Swoffer K. (2009). Critical Control Points and Operational Prerequisite Programs. Presentation from the Coca-Cola Food Safety Conference, December, Shanghai. URL: http://fskntraining.org/sites/default/files/coca-colaFS09/PRP_04_English.pdf. Accessed 4 October 2020.

13) Chen H., Liu S., Chen Y., Chen C., Yang H., Chen Y. (2020). Food safety management systems based on ISO 22000: 2018 methodology of hazard analysis compared to ISO 22000: 2005. Accreditation and Quality Assurance. 25(1): 23-37. [DOI: 10.1007/s00769-019-01409-4]

14) Chen H., Liou B.K., Chen C.S., Chuang P.T. (2020). Risk analysis method used in small-and medium-sized food enterprises implementing ISO 22000: 2018 and HACCP to conditionally determine "inspection-acceptance" as a critical control point". Accreditation and Quality Assurance. 25(5-6): 339-354. [DOI: 10.1007/s00769-020-01447-3]

15) Politis Y., Krokos F.D., Papadakis, I. (2017). Categorization of control measures in food safety management systems: the COMECAT method. British Food Journal. 119(12): 2653-2683. [DOI: 10.1108/BFJ-01-2017-0018]

16) ISO (2018). ISO 22000:2018, Food Safety Management Systems - Requirements for any Organization in the Food Chain. International Organization for Standardization.

17) Commission Notice (2016). Commission notice on the implementation of food safety management systems covering prerequisite programs (PRPs) and procedures based on the HACCP principles, including the facilitation/flexibility of the implementation in certain food businesses. The Official Journal of the European Union. C278 (59): 1-32. 


\section{Appendix}

Table X. Examples of application of COMECAT methodology to feta cheese production (Politis et al. 2017)

\begin{tabular}{|c|c|c|}
\hline \multicolumn{3}{|l|}{$\mathbf{X . 1}$} \\
\hline \multicolumn{2}{|l|}{ ISO 22000 criterion } & COMECAT methodology element \\
\hline \multicolumn{2}{|c|}{ the likelihood of failure of its functioning } & $\begin{array}{l}\text { Decision Tree, Q5 - Is it necessary to strictly monitor the } \\
\text { performance of the control measure? } \\
\text { Factors to consider } \\
\text { a) the stability of the performance of the control measure, } \\
\text { b) any synergistic effects }\end{array}$ \\
\hline \multicolumn{3}{|l|}{ Example } \\
\hline Process Step & \multicolumn{2}{|c|}{ 1. Intake of raw milk } \\
\hline Hazard identification & \multicolumn{2}{|c|}{ Presence of aflatoxins } \\
\hline Control measure & \multicolumn{2}{|c|}{ Screening tests for aflatoxins } \\
\hline \multicolumn{3}{|l|}{ Hazard assessment } \\
\hline Probability & \multicolumn{2}{|l|}{3} \\
\hline Effect & \multicolumn{2}{|l|}{3} \\
\hline Hazard significance Level & \multicolumn{2}{|l|}{3} \\
\hline \multicolumn{3}{|c|}{ Control measure Categorization } \\
\hline Initial Category & \multicolumn{2}{|l|}{ oPRP/CCP } \\
\hline \multicolumn{3}{|l|}{ Decision Tree } \\
\hline Q2 & \multicolumn{2}{|l|}{$\mathrm{NO}$} \\
\hline Q3 & \multicolumn{2}{|l|}{ YES } \\
\hline Q4 & \multicolumn{2}{|l|}{ YES } \\
\hline & \multicolumn{2}{|c|}{ NO [a) stability of the performance] } \\
\hline Q5 & \multicolumn{2}{|c|}{$\begin{array}{l}\text { Justification - Historical data, validation data and scientific literature (objective } \\
\text { evidence) demonstrate that the screening test for aflatoxins implemented, } \\
\text { exhibits a stable performance and thus the likelihood of failure of its function is } \\
\text { low. }\end{array}$} \\
\hline Categorization & \multicolumn{2}{|l|}{ oPRP } \\
\hline \multicolumn{3}{|l|}{$\mathbf{X . 2}$} \\
\hline \multicolumn{2}{|l|}{ ISO 22000 criterion } & COMECAT methodology element \\
\hline \multicolumn{2}{|c|}{$\begin{array}{l}\text { the effect on identified significant food safety } \\
\text { hazards }\end{array}$} & $\begin{array}{l}\text { Risk analysis Matrix - Probability of hazard occurrence in } \\
\text { the end product }\end{array}$ \\
\hline \multicolumn{3}{|l|}{ Example } \\
\hline Process Step & \multicolumn{2}{|c|}{ 1. Intake of raw milk } \\
\hline Hazard identification & \multicolumn{2}{|c|}{ Presence of veterinary residues (antibiotics) } \\
\hline \multicolumn{3}{|l|}{ Hazard assessment } \\
\hline Control measure & \multicolumn{2}{|c|}{ Screening tests for antibiotics } \\
\hline & \multicolumn{2}{|l|}{3} \\
\hline Probability & \multicolumn{2}{|c|}{$\begin{array}{l}\text { Justification - Failing or lacking of the specific control measure does not result in } \\
\text { the systematic presence of the hazard in the end product but the hazard can be } \\
\text { present in a certain percentage of the end product in the associated batch. }\end{array}$} \\
\hline Effect & \multicolumn{2}{|l|}{3} \\
\hline Hazard significance Level & \multicolumn{2}{|l|}{3} \\
\hline
\end{tabular}




\section{2 | International Journal of Scientific and Management Research 5(1) 1-14}

Control measure Categorization

\begin{tabular}{|l|l|}
\hline Initial Category & oPRP/CCP \\
\hline Decision Tree & NO \\
\hline Q2 & YES \\
\hline Q3 & YES \\
\hline Q4 & NO \\
\hline Q5 & oPRP \\
\hline Categorization &
\end{tabular}

X.3

ISO 22000 criterion

the location in relation to other control measure(s)
COMECAT methodology element

Risk analysis Matrix - Probability of hazard occurrence in the end product

Example

Process Step

Hazard identification

2. Cold storage of raw milk

Hazard assessment

\begin{tabular}{|l|l}
\hline Control measure & Temperature control
\end{tabular}

\begin{tabular}{|l|l}
\hline \multirow{3}{*}{ Probability } & 1 \\
\cline { 2 - 2 } & Justification - There is a next step in the production process which will eliminate
\end{tabular}
or reduce the hazard to an acceptable level (pasteurization)

Effect

1

Hazard significance Level

1

Control measure Categorization

\begin{tabular}{|l|l} 
Initial Category & PRP
\end{tabular}

X.4

ISO 22000 criterion

whether it is specifically established and applied to

reduce the hazards to an acceptable level

COMECAT methodology element

Decision Tree, Q2 - Is the control measure specifically designed to eliminate or significantly reduce the level of hazard(s) to acceptable levels?

Example

Process Step

Hazard identification

Control measure

Survival of pathogens

Temperature / time (flow) control, Divert valve control

Hazard assessment

\begin{tabular}{l|l} 
Probability & 4
\end{tabular}

\begin{tabular}{l|l}
\hline Effect & 3
\end{tabular}

\begin{tabular}{l|l}
\hline Hazard significance Level & 3
\end{tabular}

Control measure Categorization

\begin{tabular}{l|l} 
Initial Category & oPRP/CCP
\end{tabular}

Decision Tree

Q2

YES

Pasteurisation is a "killing" step, specifically designed to eliminate or significantly reduce the level of hazard(s) to acceptable levels

Categorization $\mathrm{CCP}$

Comments on Decision tree 


\section{3 | International Journal of Scientific and Management Research 5(1) 1-14}

\begin{tabular}{|c|c|c|}
\hline \multicolumn{3}{|l|}{$\mathbf{X . 5}$} \\
\hline \multicolumn{2}{|l|}{ ISO 22000 criterion } & COMECAT methodology element \\
\hline \multicolumn{2}{|c|}{$\begin{array}{l}\text { whether it is a single measure or is part of } \\
\text { combination of control measure(s) }\end{array}$} & $\begin{array}{l}\text { Decision Tree, Q5 - Is it necessary to strictly monitor the } \\
\text { performance of the control measure? } \\
\text { Factors to consider } \\
\text { a) the stability of the performance of the control measure, } \\
\text { b) any synergistic effects }\end{array}$ \\
\hline \multicolumn{3}{|l|}{ Example } \\
\hline Process Step & \multicolumn{2}{|c|}{ 10. Maturation ( $18 \mathrm{oC} / 15$ days $)$} \\
\hline Hazard identification & \multicolumn{2}{|c|}{$\begin{array}{l}\text { Survival / growth of pathogens due to improper maturation conditions (time, } \\
\text { temperature, humidity, } \mathrm{pH} \text { ) }\end{array}$} \\
\hline Control measure & \multicolumn{2}{|c|}{ Temperature control, control of $\mathrm{RH}$, control of $\mathrm{pH}$, control of production dates } \\
\hline \multicolumn{3}{|l|}{ Hazard assessment } \\
\hline Probability & \multicolumn{2}{|l|}{3} \\
\hline Effect & \multicolumn{2}{|l|}{3} \\
\hline Hazard significance Level & \multicolumn{2}{|l|}{3} \\
\hline \multicolumn{3}{|c|}{ Control measure Categorization } \\
\hline Initial Category & \multicolumn{2}{|l|}{ oPRP/CCP } \\
\hline \multicolumn{3}{|l|}{ Decision Tree } \\
\hline Q2 & \multicolumn{2}{|l|}{ NO } \\
\hline Q3 & \multicolumn{2}{|l|}{ YES } \\
\hline Q4 & \multicolumn{2}{|l|}{ YES } \\
\hline & \multicolumn{2}{|c|}{ NO [b) synergistic effects] } \\
\hline Q5 & \multicolumn{2}{|c|}{$\begin{array}{l}\text { Justification - Addition of starter cultures / rennet / CaCl2, dry salting, } \\
\text { maturation and ripening consist a multi targeted (time, temperature, salinity, } \mathrm{pH} \text {, } \\
\mathrm{RH} \text { ) combination of control measures that act synergistically in inhibiting or } \\
\text { reducing the number of micro-organisms }\end{array}$} \\
\hline Categorization & \multicolumn{2}{|l|}{ oPRP } \\
\hline \multicolumn{3}{|l|}{ X.6 } \\
\hline \multicolumn{2}{|l|}{ ISO 22000 criterion } & COMECAT methodology element \\
\hline \multicolumn{2}{|c|}{$\begin{array}{l}\text { feasibility of: } \\
\text { establishing measurable critical limits and/or } \\
\text { measurable/observable action criteria } \\
\text { monitoring to detect any failure to remain within } \\
\text { critical limit and/or measurable/observable action } \\
\text { criteria } \\
\text { applying timely corrections in case of failure }\end{array}$} & $\begin{array}{l}\text { Decision Tree } \\
\text { Q2 - Is the control measure specifically designed to eliminate } \\
\text { or significantly reduce the level of hazard(s) to acceptable } \\
\text { levels? } \\
\text { Q3 - Is it feasible to establish critical limits for the control } \\
\text { measure at this step? }\end{array}$ \\
\hline \multicolumn{3}{|l|}{ Example } \\
\hline Process Step & \multicolumn{2}{|c|}{ 5. Storage at $34-36^{\circ} \mathrm{C}$} \\
\hline Hazard identification & \multicolumn{2}{|c|}{ Growth of toxigenic bacteria if temperature abused (e.g. Staphylococcus aureus) } \\
\hline Control measure & \multicolumn{2}{|c|}{ Temperature control } \\
\hline \multicolumn{3}{|l|}{ Hazard assessment } \\
\hline Probability & \multicolumn{2}{|l|}{3} \\
\hline Effect & \multicolumn{2}{|l|}{3} \\
\hline Hazard significance Level & \multicolumn{2}{|l|}{3} \\
\hline Control measure Categoriza & & \\
\hline Initial Category & oPRP/CCP & \\
\hline
\end{tabular}


14 | International Journal of Scientific and Management Research 5(1) 1-14

\begin{tabular}{|l|l|}
\hline Decision Tree & NO \\
\hline Q2 & YES \\
\cline { 2 - 2 } Q3 \& Q4 & $\begin{array}{l}\text { Justification - For temperature control a) it is feasible to establish critical limits } \\
\text { (e.g. 34-36 }{ }^{\circ} \mathrm{C} \text { ) and b) to monitor in such a way that action can be taken in time } \\
\text { for the product to be isolated before it is used or consumed }\end{array}$ \\
\hline Q5 & NO \\
\hline Categorization & oPRP \\
\hline
\end{tabular}

